\title{
GEOBIM FOR DIGITAL BUILDING PERMIT PROCESS: LEARNING FROM A CASE STUDY IN ROTTERDAM
}

\author{
F. Noardo ${ }^{1 *}$, T. $\mathrm{Wu}^{1}$, K. Arroyo Ohori ${ }^{1}$, T. Krijnen ${ }^{1}$, H. Tezerdi ${ }^{2}$, J. Stoter ${ }^{1}$ \\ ${ }^{1}$ 3D Geoinformation group, Delft University of Technology, Delft, The Netherlands - \\ (f.noardo, t.wu, k.ohori, t.f.krijnen, j.e.stoter)@tudelft.nl \\ ${ }^{2}$ Municipality of Rotterdam, Rotterdam, The Netherlands — h.tezerdi@ rotterdam.nl
}

KEY WORDS: Digital building permit, regulations interpretation, BIM, GeoBIM, Industry Foundation Classes, data integration

\begin{abstract}
:
Among the digitalization processes which are being raised in Europe and in the world, the building permit process is seen as one of the priorities by municipalities, governmental institutions and standardization organizations. However, in current practice, the building permit issuing as well as the integration of geoinformation with BIM (GeoBIM) suffers from a number of complex subissues. These issues still remain and prevent the development of successful methodologies. In this paper, the building permit use case is explored within a project in close collaboration with the municipality of Rotterdam. A very specific case study in Rotterdam was selected as a starting point, which allowed us to develop the needed methodology for the implementation of an effective tool. In this paper we highlight the interpretation and formalization of regulation for building height, overhang and tower ratio. While these rules are specific to a zoning plan in Rotterdam, we believe that the methodology and encountered issues in formalizing the rules, applying the rules on delivered models and integrating various data sources (BIM and GIS specifically) are general to most building codes.
\end{abstract}

\section{INTRODUCTION}

With the development of 3D model-related technologies, especially regarding the design of buildings with Building Information Models (BIMs), and the improvement of digital and web systems, the advantages of automated building permits issuing systems became apparent in many countries. The process can be faster and more objective. In addition, errors and misunderstanding in the design evaluations and in the interpretation of regulations can be avoided, bringing benefits (included economic) from the reduced amount of needed resources. Furthermore, the built environment can benefit from an automated system, since more consistent decisions can be taken considering more complex parameters (for example, the whole area can be considered in $3 \mathrm{D}$, instead of having $2 \mathrm{D}$ or partial views, which are also limited to what is delivered by applicants for building permits). And a quick turnaround of model check outcomes may result in a more iterative process and a higher quality built environment. Attempts in such a direction are being developed in many countries, starting perhaps with the CORENET ePlanCheck project in 1995 in Singapore and later in Europe ${ }^{1}$ (Noardo et al. 2019b) and in the rest of the world. Some studies proposed methods and tools for checking the building regulations by means of BIMs (e.g. Kim et al. (2016); Getuli et al. (2017); Choi and Kim (2017); Plazza et al. (2019)) and others considered the integration of BIM and 3D city models (e.g. in Sweder ${ }^{2}$.

\footnotetext{
* Corresponding author

1 e.g. Sweden, Finland (http://www . aecbytes.com/feature/200 5/CORENETePlanCheck.html https://kirahub.org/en/home/) some municipalities (like Vantaa) in particular, Estonia (https ://ae c-business.com/digital-transformation-of-the-estonia n-construction-sector-an-interview-with-jaan-saar/, Germany, United Kingdom (https : //www.cdbb.cam.ac.uk), some of which are described in the presentations at https://kirahub.or g/en/wdbe2020/pre-event-2/\#presentations

2 https://www.smartbuilt.se/in-english/
}

A common issue in those previous experiences is taking into account the small specific parts of the process and the challenges given by the use of involved data in a consistent way. Especially when standardised information is involved, as it is sensible for fostering interoperability and re-usability, some further issues could arise, also given by the nature of standards themselves (e.g. Noardo et al. (2020a)). To push even further this interest, the European Directive 2014/24/EU ${ }^{3}$ was published, strongly encouraging the use of BIM for public projects. The result of this is that in many countries from 2018 a process began towards the mandatory adoption of BIM, at least for public buildings, generally to be fulfilled by 2022 .

The organizations responsible of single portions of the topic are addressing it internally (for example, see the buildingSMART effort in its Regulatory Room 4 . However, a synergy of disciplines, expertises and stakeholders are needed to face such multifaceted and complex issue in a thorough way. This goal is being pursued from beginning 2020 within the European Network for Digital Building Permit (EUnet4DBP) ${ }^{5}$ (Noardo et al. 2020b).

\subsection{The premises to this work: The EuroSDR GeoBIM project and Rotterdam digital city}

Furthermore, stakeholders from many European countries, participated in the EuroSDR GeoBIM project $(2017-2020)$ aiming at the the development of a coherent approach to the integration of geoinformation with BIM with consensus between multiple

\footnotetext{
https://eur-lex.europa.eu/legal-content/EN/TXT/?uri =celex\%3A32014L0024

4 https://www.buildingsmart.org/standards/rooms/regula tory/

5 https://3d.bk.tudelft.nl/projects/eunet4dbp/

6 https://3d.bk.tudelft.nl/projects/eurosdr-geobim/ (The Netherlands, Ireland, UK, France, Spain, Switzerland, Slovenia, Poland, Denmark, Finland, Sweden, Norway are involved)
} 
stakeholders from both the geoinformation and the BIM sides, working at an international level. The automation of the building permit issuing was one of the use cases of the project (the other being asset and facility management).

In order to provide scalable and shareable methods and tools, international open standards, fostering interoperability were chosen as reference data formats. Therefore, CityGML ${ }^{7}$ by the Open Geospatial Consortiumand the Industry Foundation Classes (IFC) ${ }^{8}$ by buildingSMARTwere chosen.

The investigation approach in the project, where national mapping and cadastral agencies (NMCAs), together with some research institutes participated, was mainly guided by the application, starting the investigation with interviewing municipalities operators and analysing current practice workflows (Noardo et al. $2019 \mathrm{a}{ }^{9}$ The collaboration with the municipality of Rotterdam started within this context.

Meanwhile, the Rotterdam "Digital city" pilot is on-going, as the effort of the Rotterdam Municipality to transpose procedures to a digital integrated environment, able to help the optimization of resources and improve the efficiency of management, through integrated information, city monitoring and automatic processes. A part of this is the work towards automation (or semi-automation) of the building permit process, for which a specific project was funded to investigate the specific cases with available data and develop a demonstrator able to be extended and scaled as a support to the building regulation checks.

\subsection{Objectives}

This paper firstly describes the methodology followed to develop the project by starting from concrete cases and data by designers. As a necessary preliminary step, the interpretation and formalization of the considered regulation is explained. In addition, the observations about the use of the geometry represented in the BIM to support the checks of such regulation are outlined.

\section{METHODOLOGY}

Although a huge discussion is on-going about the building permit automation topic, concrete, complete, flexible and scalable solutions are seldom implemented, and remaining issues are still widespread. The methodology we followed was therefore built step by step, with previous steps enabling the unveiling of useful following ones. The big challenge in such a use case, besides the multidisciplinarity, is the little development, with respect to systematic adoption of criteria and methods to produce and store information, of almost all the premises intended to support their final synergy for checking urban regulations (regulations text, the zoning maps, the 3D city model, the BIMs, the checking procedures).

The approach we adopted was therefore completely bottom-up, starting from the previous investigations and interviews performed within the EuroSDR project, to proceed step by step in

\footnotetext{
7 http://www.citygmlwiki.org

8 http://www.buildingsmart-tech.org/specifications/if c-overview

9 A harmonised workflow was developed and reviewed by the partners and many municipalities in different countries (https://3d .bk.tudelft.nl/projects/eurosdrgeobim/Workflow Description.pdf) EuroSDR GeoBIM project - Integrated Workflow using GeoBIM information for building permit process. Zenodo. http://doi.org/10.5281/zenodo.3948493
}

collaboration with the Municipality officers and from the available data sets.

First step was the selection of 2 specific regulations among the ones that were deemed likely to have the best advantage from GeoBIM: building dimensions and parking places. In this paper the results regarding the dimensions regulation are described. Those regulations were selected, in consultation with Municipality experts, because these were judged to be among the simplest ones to implement. After solving the most straightforward issues for these cases, the work could be further extended more easily.

Moreover, those two regulations were expressed for one specific zone, where a case study was chosen. The selected case study (Section 2.1) lies in the centre of Rotterdam, in the Maritiem district, where building works are ongoing and recent submissions of building permits were just approved, following the most up-to-date approval procedure.

The specific text of the regulation was analysed with the aim of translating it to a formal language that would be able to be interpreted by machines. Several studies propose methods to automatically interpret the natural language of regulations transforming them to code to check the proposed building design, as represented in models (e.g. the UK D-COM activity ${ }^{10}$. However, challenges appeared long before the conversion of this information from natural language to any formal language, since even for humans, the interpretation of the regulations' text can be open to a number of different interpretations (see Section 3.1), whilst an effective code should be absolutely unambiguous.

A relevant step to solve this was the organization of a specific workshop by the Rotterdam Municipality (H. Tezerdi and R. Manbodh), with the involvement of expert operators usually checking and deciding on building permits themselves, who know by experience how to interpret the main building regulation (the bouwbesluit). The details and meanings of the two chosen regulations were explained and agreed upon and the specific questions we had about ambiguous statements were answered. Some more details arising during the following work towards implementation were asked later. That was the only way to have ambiguities about the regulation solved. This issue already showed how little the current state of regulations lends itself to automation. After that, the regulation could be formalised step by step and the related information was extracted from where it was deemed most effective (either in the BIM, in the 3D city model or elsewhere). Only at this point, it was possible to define the sub-issues to be tackled, now appearing sharper. These ones, with related research questions can be listed as:

BIM, and specifically IFC, georeferencing How the georeferencing information is currently attached to BIM and how it would be most useful? How to help designers adding georeferencing to BIM? How to make sure it is stored in the correct place in the IFC?

Extraction of the building envelope What elements of the BIM should be considered? Is it possible to use only one of the several IFC models composing the BIM? Can we consider spatial structure segmentations, such as storeys and bounding boxes, for approximating the envelope? How can we choose the reference surface and what tolerances

\footnotetext{
${ }^{10}$ http://www.dcom.org.uk
} 
do we consider for generalising the building envelope in a suitable way? And finally, what algorithms can we use and what kind of geometry can be most effective as as input/output?

Building envelope segmentation Where should the building envelope be segmented to support the checking of the regulation? What tolerances are to be considered? How to automatically detect the different segments?

\section{Measuring the model to check regulations, using GeoBIM} How to obtain the starting point for measuring heights in the $3 \mathrm{D}$ city model? How to measure the overlapping area of the two detected parts? How to automatically detect the directions "towards Boompjes" or "towards Heterkade" from the integration of BIM and 3D city model?

As a base to start addressing these issues in a proper way, we should necessarily analyse and work with the specific involved data, besides looking at the standard data models. In fact, since there are few specific prescriptions and criteria about how to model and store specific elements in IFC, we cannot exactly know what to expect from such models. This degree of freedom could be useful for adapting to different use cases, but actually hinders interoperability, allowing a set of alternatives which are too open for practical purposes. Exactly the same issue is found for CityGML (e.g. where is the height of LoD1 building model?).

Furthermore, other features of the BIM, usually composed by several IFC files, depend on the building design practice itself, and can be different from what an outside person can imagine. For 3D city models, the field is a bit more restrained, but equally open to different choices, which remain unknown, unless good information is available as metadata about modelling choices that were made and reference datasets that were used.

The solution to the permissiveness of IFC and CityGML could be to make the standard itself to be more restrictive, through specific guidelines, or possibly by developing tools to assist in the preparation of the models. The guidelines formulated in this project will be used to rule the modelling of BIMs and 3D city models for the use case. For these reasons, the two examples of BIMs representing the designed, mainly residential, buildings, with a quite good quality, were a great advantage to guide the choices during implementation. They were preventively manually inspected, in order to both understand how to effectively use them and to verify and measure the ground truth, useful to assess the result.

\subsection{Case study}

In consultation with the Municipality of Rotterdam, we selected the zone "Centrum 3" of the Waterstad bestemmingsplan (destination plan) in the centre of Rotterdam (Maritiem district). Within the area, two recently designed buildings were used as the case study for this project: the so-called Peak tower (Figure 1) and the Terrace tower (Figure 2). The two available BIMs were kindly provided in IFC (v.2x3) for the tests.

The Peak tower is composed by structural, architectural and facades models. They are correctly registered together, by means of the same reference point and orientation. They are not georeferenced, though: the reference point coordinates are $(0,0,0)$, whilst the attributes in the IfcSite would locate the model in a general location in Amsterdam.
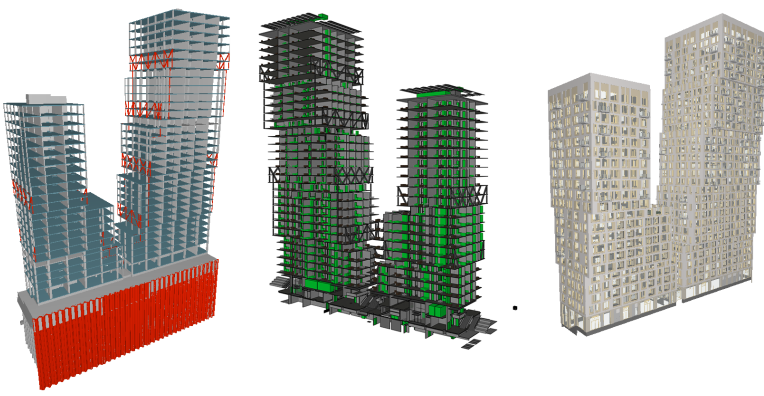

Figure 1. The three IFC models composing the Peak tower BIM, from left to right: structural, architectural and facades.

The Terrace tower BIM is represented by structural and architectural IFC models, plus one representing the context and elements probably belonging to the work site. They are correctly registered together too, and in this case they are also georeferenced by storing projected coordinates in a point related to the IfcSite, according to a level of georeferencing LoGeoRef 20 (Clemen and Hendrik, 2019).

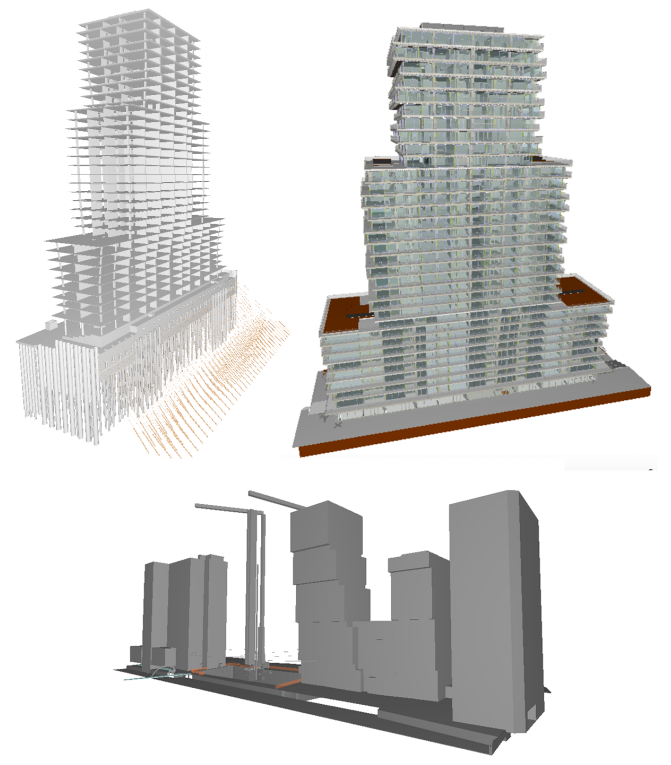

Figure 2. The three IFC models composing the Terrace tower BIM, clockwise, the structural, the architectural and the context one.

For the integration, georeferencing is essential. In order to georeference these specific models properly, we followed a complex manual process, passing through the representation of the model in the city context as delivered in PDF, measuring there the coordinates of common points in the city map and in the BIM plan. This process was successful, but it is definitely not a procedure to be preferred. A reliable solution will be developed with guidelines and assisting reference data and tools.

\section{RESULTS}

\subsection{Regulations interpretation}

The regulation considered here is the Waterstadt - Artikel 5 Centrum - 3, specifically, the section 5.2 Building rules - 5.2.3 Building standard 11

\footnotetext{
11 https://www.ruimtelijkeplannen.nl/documents/NL . IMRO. 0599.BP1054Waterstad-va01/r_NL. IMR0.0599.BP1054Waters
} 
The text, here translated to English, is: "The maximum building height is 100 meters, on the understanding that it can be realized with a substructure of a maximum of 17 meters high and a construction of a maximum of $50 \%$ of the surface of the substructure. At the location of Boompjes 60-68 and Boompjes 55-58, an overhang of 5 meters on the Boompjes side and 10 meters on the Hertekade side is permitted".

Although being a very short text, when considering it for formalization, several ambiguities and uncertainties arise, also for human interpretation. For example:

- "The maximum building height is 100 meters" - Where is the 0 to be considered for starting measuring? What elements have to be counted (e.g. if installations or antennas rise above, do they count?)

- "on the understanding that it can be realized with a substructure of a maximum of 17 meters high and a construction of a maximum of 50\% of the surface of the substructure."- Do the substructure has a minimum height? If the substructure or the top structure are split in different parts (like multiple towers), do they count as one? Is it allowed? Must the substructure cover the whole parcel? Is any alignment to be considered? Is the footprint or the outline of the top part to be considered for measuring the overlap? Is the $50 \%$ including only the overlapping footprint of the top part or also the area of possible overhangs?

- "At the location of Boompjes 60-68 and Boompjes 55-58, an overhang of 5 meters on the Boompjes side and 10 meters on the Hertekade side is permitted"- How do we detect such locations (are they parcel names, addresses, what else)?

Those were mainly solved during the organised workshop on the 18th October 2019 in Rotterdam Municipality, with the help of the expert Rotterdam municipality officer knowing the rules and performing the checks. Later, further ambiguities could be solved thanks to the continuous collaboration with the Municipality, which was critical for the success of this initial phase.

\subsection{Regulation formalization}

To allow a more easy conversion to a machine-readable code, the rules stated by the regulation were written in a more formal language according to explanation.

First, the building $(B)$, intended as a whole, has to be considered as the composition of two superimposed parts, i.e. bounding volumes ( $B P 1$ and $B P 2)$, formally defined as $B:=$ $B P 1 \cup B P 2$, or more in detail:

$$
B P 2 x y \cap B P 1 x y \quad A N D \min (B P 2 z) \leq \max (B P 1 z)
$$

Moreover, $B P 1$ and $B P 2$ can possibly consist of several disconnected parts, therefore we should consider:

$B P 1=B P 1 a \cup B P 1 b \cup \ldots \cup B P 1 n$
$\frac{B P 2=B P 2 a \cup B P 2 b \cup \ldots \cup B P 2 n}{\text { tad-va01.htm1\#_5_Centrum-3 }}$

Eventually, it is necessary to consider a different segmentation of the building, in which the portions of $B P 2$ overhanging from $B P 1$ are detected for calculating the maximum overhang towards the two streets (Boompjes and Heterkade) (Figure 3 .

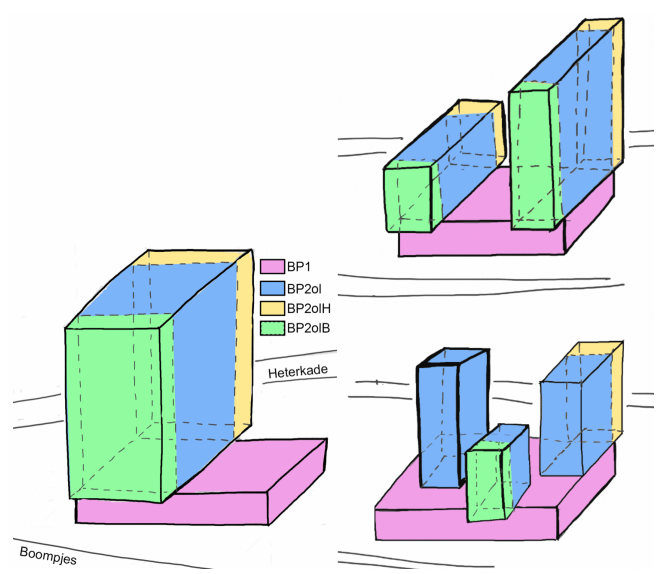

Figure 3. Building segmentation to check overlap percentage and overhangs dimensions, where $B P 2 o l$ is the part of $B P 2$

with a footprint overlapping the $B P 1$ one; $B P 2 o h B$ and

$B P 2 o h H$ are the $B P 2$ parts with outline overhanging, towards

"Boompjes" and "Heterkade" streets respectively.

$B P 2=B P 2 o l \cup B P 2 o h B \cup B P 2 o h H$

Once defined the different parts we are able to check the dimensions according to the regulation.

The ratio between the footprin ${ }^{12}$ area footprint. A of the segment of $B P 2$ overlapping $B P 1$ (i.e. $B P 2 o l$ and the total $B P 1$ area, has to be lower than $50 \%$. That is:

$$
\text { footprint. } A(B P 2 o l) \leq 0.5 \times \text { footprint. } A(B P 1) \text {. }
$$

Moreover, the total building height needs to be lower than 100 $\mathrm{m}$, with starting height according to the rules:

- for a structure, the main entrance of which is adjacent to the road: the height of the road at the top of the road;

- for a building whose main access is not adjacent to the road: the height of the site at the location of that main access, after completion of the construction of that site;

- if a structure is built on more than one road, the level of the highest road is the norm.

Moreover, the minimum height for $B P 1$ should be $2.6 \mathrm{~m}$, which is not a number explicit from this regulation, but it is implied, since being the minimum ceiling height defined by bouwbesluit (the Dutch building codes). We therefore obtain the rule to be checked:

$$
2.6 m \leq \operatorname{Height}(B P 1) \leq 17 m \text {. }
$$

Finally the maximum outline overhanging of the top part $B P 2$ with respect to $B P 1$, in the two directions has to be measured.

\footnotetext{
12 'footprint' is the part touching and intersecting the ground (or the building part below); 'outline' of the building is the maximum extension on $x y$ plane of the maximum envelope of the building, including overhanging parts and $3 \mathrm{D}$ extension.
} 
We need the information about the parcel address (Boompjes 55-58 and Boompjes 60-68), for which we should rely on an attribute in the models. We will come back to this when defining suitable guidelines for (3D city model) modellers.
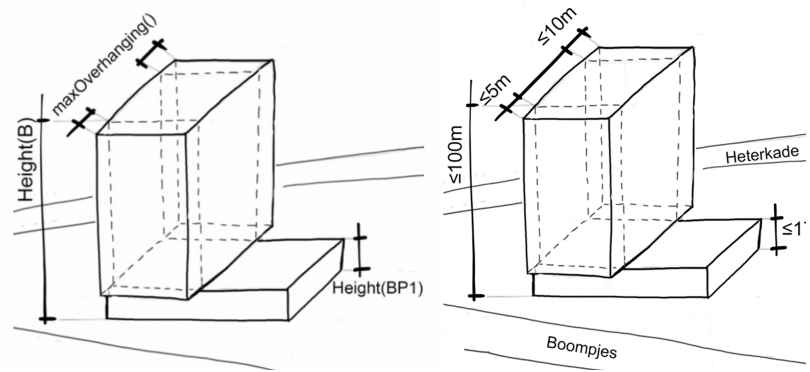

Figure 4. Dimensions to be checked.

After these detailed definitions, it was possible to begin the work addressing the specific sub-issues involved in the solution of each part (Sections 3.3 - 3.5.

Moreover, it is essential to understand the treated data and where the needed information could be obtained, therefore, an inspection of the models was performed to guide the implementation and guidelines proposal.

\subsection{Building envelope extraction}

For the dimensions regulation check, we need to consider the envelope of the building. It would be possible to ask the architect to generate it before submission (and trust the information that is provided), but it is preferable if it is automatically generated from the BIM. Since BIMs are usually split in several models, it is necessary to consider all of them together. Moreover, to approximate the shape of the building two solutions could be used:

- minimum (rotated) bounding box ( $B B B)$, to understand the orientation w.r.t the 3D city model (e.g. the regulations make reference to street names), maximum height;

- building envelope $(B E)$, to calculate dimensions.

However, the extraction of such geometries is not straightforward, since a generalization has to be applied to a very complex surface (even though this facade can appear mostly flat from afar), and a selection of discontinuity is necessary, probably according to a tolerance, to obtain a suitable envelope (Figure 5).

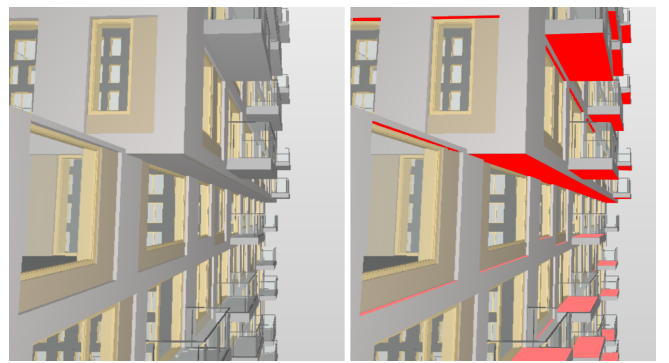

Figure 5. Close look at the facade: horizontal planes (in red) can all be potentially read as discontinuities of vertical surfaces.

Alternative approaches can be followed for this task, such as using storey attributes ${ }^{13}$ 2D alpha shapes, 3D alpha shape, and voxelization.

13 e.g. https://gist.github.com/aahoo/c97248816510bf 9892 a2e8bdf90d1626
Once this is done, we can proceed to the envelope segmentation or measurement, as useful to check the regulations. Alternatively, the minimum bounding box of the building can be calculated from the total envelope if necessary.

3.3.1 Building local systems Before going to the building envelope segmentation, a reasoning about the different kind of needed Cartesian systems is necessary. $O^{M}$ is the origin of the $\mathrm{BIM}$ and to which the georeferencing information is associated. In the case of very extended models, more than one $O^{M}$ will be provided: in that case the model will be split in the different parts, having one $O^{M}$ each. In order to segment the building and check the rules as stated in the regulations (heights, distance, overhangs and so on), it is necessary to refer to different local building Cartesian systems. The local Cartesian system used during the building design is chosen by the architect or engineer according to the design requirements. Therefore it is not straightforward that it is also the best needed for building permits calculations (Figure 6).

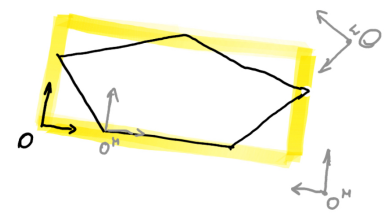

Figure 6. CRS for the checks calculations ( $O$, in black) and possible others CRS $O^{M}$ possibly used to attach georeferencing information. In yellow, the bounding box of the building.

We can instead set two more local Cartesian systems to be used in the calculations (Figure 7):

- The origin $(O)$ of the first one (LocalTotal) can be set in one of the lowest vertexes of the bounding box, with the $x$ and $y$ axis positive directions aligned to the bounding box base and the $\mathrm{z}$ axis upward.

- The second one (LocalOutside) is parallel to LocalTotal, but it is translated upward along the $z$ axis, until the origin $\left(O^{I}\right)$ leaves at the intersection of the building bounding box with the terrain, for example according to the rules stated in Section 3.2
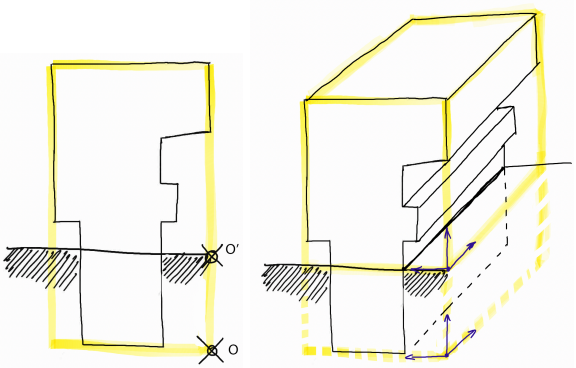

Figure 7. The origin of the two Local systems useful for regulation checks: LocalTotal, with origin in $O$ and LocalOutsite with origin in $O^{i}$.

\subsection{Building envelope segmentation}

Again, a suitable method to detect horizontal discontinuities in the building envelope has to be chosen. 
After that, it is possible to segment the building envelope accordingly: cut the building envelope in as many parts $(P 1, P 2$, $\ldots, P N, \ldots, P M)$ as the horizontal discontinuities are (Figures 89.

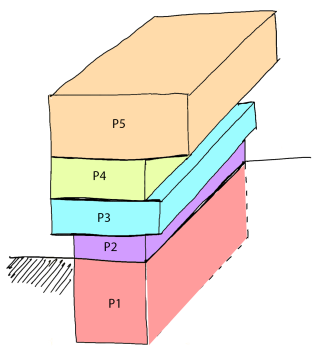

Figure 8. Segmentation of the building envelope according to the horizontal discontinuities.
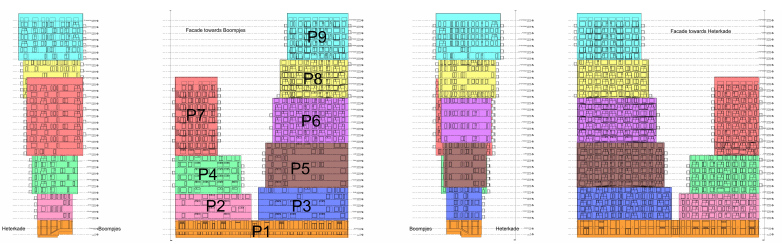

Figure 9. Segmentation of the building envelope of the actual BIM according to the horizontal discontinuities.

In the LocalOutside Reference system:

IF $\quad P 1_{z \max } \leq 17 m \quad A N D \quad P 2_{z \min } \geq 2.6 m$
$T H E N \quad B P 1=P 1$
ELSEIF $\quad P 2_{z \max } \leq 17 m \quad A N D \quad P 3_{z \min } \geq 2.6 m$

$$
\begin{gathered}
\text { THEN } \quad B P 1=P 1+P 2 \\
\text { ELSEIF } P N_{z \max } \leq 17 m \quad A N D \quad P N+1_{z \min } \geq 2.6 m \\
\text { THEN } B P 1=P 1+P 2+\ldots+P N
\end{gathered}
$$

on the same level are contiguous (they are a unique building part) or they are separated in more than one part.

For the segmentation, we should exclude the facade articulations (overhangs and recesses) being smaller than a tolerance. For example, almost at the border between $P 1$ and $P 2$, but with this segmentation, completely fitting within $P 2$, there is a small change in the facade surface, protruding $8 \mathrm{~cm}$ more towards the exterior in the top part. However, this is a negligible change, not worth a cut in the building. The further protruding or recessing parts of the building, that deserve to be considered as vertical discontinuity, are in the order of approximately $3.5 \mathrm{~m}$. Therefore, we could set the tolerance to either an absolute value (e.g. 1 meter, that would measure $0.5 \mathrm{~cm}$ in a scale $1: 200$ paper drawing, or 0.5 meter to be more inclusive), or maybe to a percentage of the building horizontal extent (for example 5\% or $10 \%$ ). This should be further discussed with the municipality officers.

An approximated option could be considering the difference between storeys footprint (or overlay) coordinates, possibly choosing a threshold to assess if such a difference is worth the detection of a discontinuity. For example, a percentage with respect to the total lenght of a floor could be chosen (e.g. if the difference is less than $5 \%$ they are part of the same segment).

However, to rely on one footprint per storey would lead to the exclusion of possible protruding parts above or below the section height, although it can be useful to intentionally exclude specific objects, such as balconies.

This opens up the discussion to a further point, i.e. the possible selection of the elements to be included or not in the relevant envelope. The balconies are a typical case.

\subsection{Checking the building dimensions}

At this point, it would be possible to check the regulation, split in its different parts.

First, it has to fit in the parcel. Therefore, considering the BP1 envelop in the georeferenced system:

\section{ELSE

$$
\text { --NOTCOMPLIANTTOREGULATION }
$$

$\mathbf{B P 2} \equiv$ TotalBuildingEnvelope $-B P 1 \equiv P(N+1)+\ldots+$ $P M$

Looking at the BIM representing the designed building, we can observe that $P I$ is the only part extending for the whole building, below both the towers leaned on the top of it. In the orange part of Figure 9 representing $P 1$ is $6.89 \mathrm{~m}$ high, as measured in the part where the discontinuity with the top parts is visible, and not including the whole ground storey. That would be a bit higher, but is not reflected in the building sections. The following part, $P 2$ is $16.86 \mathrm{~m}$ high Therefore, it could be considered as part of the base. However, $P 3$, which begins at the same level, raises up to $19.9 \mathrm{~m}$, which is more than the allowed 17 meters.

Since in this case the parts are not piling up one on the top of the other one, it is more difficult to check the rules with the previous equations. The easiest and probably most sensible choice in this case could be to separate the towers from the base. Therefore, we can consider $P 1$ as $B P 1$ in this case and the two towers being $B P 2$. In similar cases, the software should be able to recognize when all the building parts (and, consequently, elements)
- georef MaxOutlineBP $1 \subseteq$ georef MaxOutlineParcel

Second, the overlapping area of the two building parts is given by:

\section{BP2bottom faceAreaoverlapping $B P 1 \leq$}

\section{$0.5 *$ AreaBP1topface}

Finally, we should calculate the maximum height (in the LocalOutside Reference system: BP2_zmax $\leq 100 \mathrm{~m}$

The tolerances to the rule can be considered 0.5 meters (in the case it is required for an architecturally responsible rounding off the top construction layer or roof / sloping roof surface) considering the Article 19.1 "General derogation rules - Deviation from permitted architectural height, whilst if the building height exceed of no more of 5 meter, the system could give a warning for the case being assessed by a commission possibly deciding for the exemption allowed by the Article 19.2 "General derogation rules - Deviation from permitted construction height technique, 14 The city objects useful for the calculation

\footnotetext{
14 https://www.ruimtelijkeplannen.nl/documents/NL. IMRO. 0599.BP1054Waterstad-va01/r_NL.IMR0.0599.BP1054Waters tad-va01.html\#_19_Algemeneafwijkingsregels
} 
are provided in the 3D city model and other possible geoinformation, eventually modified according to the elements provided in the BIM model (e.g. IfcSite). We assume that if the terrain is considerably changed, the design is provided in the BIM, having borders correctly aligned with existing, 3D city model, so that it could be easily substituted without complex and pointless data fusion processing. As measured in Revit, the maximum height, considering the entrance door towards Boompjes is $103.47 \mathrm{~m}$ and $106.20 \mathrm{~m}$ starting from the entrance towards Heterkade. Considering the BIM design, the last storey is completely over the limit. However, it is entirely dedicated to installations; for this reason the exemption foreseen by the Article 19.2 can be applied and the design be approved anyway, with the 0 should be set at the entrance towards Boompjes, which is the highest one. Actually, one more check should be necessary, considering the road elevation adjacent to such entrance, instead of the entrance itself.

3.5.1 Calculating overhangs Several steps are finally necessary for the measurement of overhangs. First, it is necessary to find out if the parcel is Boompjes 60-68 or Boompjes 55-58. Considering the $B P 1$ envelop in the georeferenced system, a corresponding attribute value of the parcel within which it lies can be read (assuming that this information is stored there), determining if the rule applies.

The second step is finding out which are the faces (out of the six of the oriented bounding boxes) in the direction 'towards Boompjes' and which 'towards Heterkade': Considering the georeferenced oriented bounding boxes of $B P 1$ and $B P 2$, find, for each of them, the faces $B P X t B$ and $B P X t H$ by calculating, as alternative options:

- For each $P(P 1, P 2, \ldots, P N, \ldots, P M)$ which is the face whose normal positive direction meets each of the two streets (or virtual continuation of them);

- Intersection between a line by FaceCenter+FaceNormal intersected (2d) with wall axis and distance with FaceCenter.

In this way we find which is the face oriented towards each street for each part: $P 1 t B$ ( $P 1$ face towards Boompjes), $P 2 t B, \ldots, P N t B, \ldots, P M t B) P 1 t H$ ( $P 1$ face towards Heterkade), $P 2 t H, \ldots, P N t H, \ldots, P M t H$ ) (Figure 10.

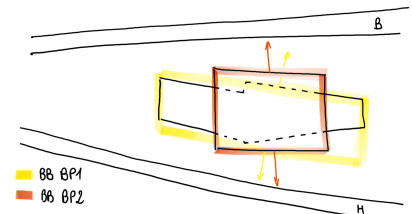

Figure 10. $B P 1$ and $B P 2$ bounding boxes, normals and streets.

Third step is the measurements of the overhangs.

We consider the oriented bounding box of $B P 2$ and the oriented bounding box of $B P 1$ in the LocalTotal or the LocalOutside reference system.

$B P 1 t B, B P 2 t B$ and $B P 1 t H, B P 2 t H$ define the maximum extension of $B P 1$ and $B P 2$ toward Boompjes and toward Heterkade respectively; $B P 1 P$ is each $P$ included in $B P 1$.

The calculation of $B P 1 t B$ (and similar for the other parts $B P 2 t B, B P 1 t H, B P 2 t H)$ is:
IF Boompjes is met in positive direction of LocalOutside CRS y axis

$$
\text { THEN } \quad B P 1 t B=B P 1 P t B y \cdot \max { }^{15}
$$

ELSEIF Boompjes is met in negative direction of LocalOutside CRS y axis

$$
\text { THEN BP1tB }=\text { BP1PtBy.min }
$$

ELSEIF Boompjes is met in positive direction of LocalOutside CRS $x$ axis

$$
\text { THEN } B P 1 t B=B P 1 P t B x \cdot \max
$$

ELSEIF Boompjes is met in negative direction of LocalOutside CRS $x$ axis

$$
\text { THEN BP1tB }=\text { BP1PtBx.min }
$$

Now the overhang towards Boompjes can be checked:

$|B P 1 t B-B P 2 t B| \leq 5 m$

Considering the ground truth as measured in Revit, the maximum overhang towards Boompjes is $5 \mathrm{~m}$, therefore, the regulation should result as approved.

And the same towards Heterkade:

$|B P 1 t H-B P 2 t H| \leq 10 m$

Considering the ground truth as measured in Revit, the maximum overhang towards Heterkade is $9 \mathrm{~m}$, therefore, the regulation should result as approved.

\subsection{The resulting tool}

The explained concepts guided the initial implementation of a too ${ }^{16}$ that starts from the IFC files to support the checks. Other issues had to be tackled before arriving to the implementation, but the work described in this paper was the essential starting point for the tool to be effective with real-world data about design-related BIM and regulations.

\section{DISCUSSION}

The issue of automatic regulation checking appears as a series of nested challenges, which can only be detected or disclosed if very closely investigated. For this reason, the choice of proceeding from a specific case study revealed to be a fruitful approach, even though at first sight is could seem lacking scalability. For example, other regulations will present different challenges; notwithstanding this, the reflection about how to consider the geometry represented in the BIM and the solutions found for extracting it or its relevant parts is a step likely regarding a big part of the cases, to which the solutions can be adapted after little changes in the otherwise solid procedure. The representation of geometry in the BIM will be very similar in all the cases as well. It makes, for example, the reasoning about the necessary generalizations relevant (e.g. what are the tolerances to be considered when distinguishing between different elements or parts of the building).

\footnotetext{
15 i.e. The maximum y value of the faces detected towards Boompjes among all the parts composing BP1.

16 https://github.com/twut/GEOBIM_Tool
} 
The resulting single detailed steps could appear as more challenging than a simplified but faster solution (e.g. checking if the building fits into a box having maximum dimensions). However, solving this actually makes the basis for scaling up the project, and it shows how such systems can actually align with the municipality current practice. In addition, the strict collaboration with municipality officers allows correct results to be obtained and should be a central part of any similar study.

Beyond the limitations of this study, some more general challenges are present, which were partially pointed out in this paper: first of all the ambiguity in regulations, the difficulty in their interpretation and the existence of several different regulations about similar parameters, coming from different fields. The still suboptimal interoperability of BIM models (and other involved geoinformaton) is another issue, due to the implementation of standards, standards themselves and followed best practices.

Although such hindrances are present, the automation of at least some steps of the building permit process would allow a higher efficiency in the planning procedures: the saved resources could be effectively re-invested in the current flaws of the system (e.g. difficulty in managing the high number of requests, careful inspection of the most tricky or borderline cases, management of exemptions, focus on the cases where human judgement is necessary).

The implemented demonstrator could be further refined, for example to overcome the lacks and inaccuracies in the models, but it already represents a good base to deal with the information in the IFC files.

\section{CONCLUSIONS}

The paper presents the work developed for implementing a tool and a methodology able to support the municipality of Rotterdam in building permits regulation checks by means of digital solutions, starting from digital standardised datasets (mainly BIM in IFC). The approach was completely bottom-up, starting from a very specific case study (one regulation and few datasets). This allowed the explication of several sub-issues which need to be overcome before solving the most apparent steps in the building permit workflow. Addressing them could allow the formulation of guidelines for all the involved data providers (regulations, BIM, 3D city models and so on) and the implementation of a demonstrator, still in an initial state, but promising to be improved and scaled for actually supporting the municipality in the building permit task.

Future directions regard the specific analysis of the available IFC files to understand specific features, current practices, frequent limitations or inaccuracies. This will guide safer choices while implementing the tool. In addition, guidelines could be provided to produce more suitable models and avoid inconsistencies or inaccuracies. In addition, alternative algorithms could be tested for various steps, such as the detection of the most useful exterior facade geometry to support the checks. The extension of the research and tool to the check of other regulations or to other parts of the city would be an additional interesting work, as well as a wider collaboration with planners, municipality officers and designers.

\section{ACKNOWLEDGEMENTS}

This project was funded by the Municipality of Rotterdam (resp. Hasim Tezerdi and Rosen Manbodh). This project has also received funding from the European Research Council (ERC) under the European Union's Horizon2020 Research \& Innovation Programme (grant agreement no. 677312, Urban modelling in higher dimensions, and Marie Skłodowska-Curie grant agreement No. 707404, Multisource Spatial data Integration for smart City Applications).

\section{References}

Choi, J. and Kim, I., 2017. Development of rule-based building code compliance checking system for bim-based quality improvement. International Information Institute (Tokyo). Information 20(4B), pp. 2929-2936.

Clemen, C. and Hendrik, G., 2019. Level of georeferencing (logeoref) using ifc for bim. Journal of Geodesy (10), pp. 1520.

Getuli, V., Ventura, S. M., Capone, P. and Ciribini, A. L., 2017. Bim-based code checking for construction health and safety. Procedia engineering 196, pp. 454-461.

Kim, I., Jang, J. and Choi, J., 2016. Development of prespecification for bim-based automated building code checking. Korean Journal of Computational Design and Engineering 21(1), pp. 31-41.

Noardo, F., C. Ellul, C., Harrie, L., Overland, I., Shariat, M., Ohori, K. A. and Stoter, J., 2019a. Opportunities and challenges for geobim in europe: developing a building permits use-case to raise awareness and examine technical interoperability challenges. Journal of Spatial Science.

Noardo, F., Ellul, C., Arroyo Ohori, K., Biljecki, F., Harrie, L., Krijnen, T., Kokla, M. and Stoter, J., 2020a. The ISPRSEuroSDR GeoBIM benchmark 2019. In: ISPRS Congress 2020, ISPRS - International Archives of the Photogrammetry, Remote Sensing and Spatial Information Sciences, ISPRS.

Noardo, F., Ellul, C., Harrie, L., , Devys, E., Arroyo Ohori, K., Olsson, P. and Stoter, J., 2019b. EuroSDR GeoBIM Project a Study in Europe on how to use the Potentials of BIM and Geo Data in Practice. In: 14th 3D GeoInfo Conference 2019, ISPRS - International Archives of the Photogrammetry, Remote Sensing and Spatial Information Sciences, Vol. XLII-4number W15, ISPRS, pp. 53-60.

Noardo, F., Malacarne, G., Mastrolembo Ventura, S., Tagliabue, L., Ciribini, A., Ellul, C., Guler, D., Harrie, L., Senger, L., Waha, A. and Stoter, J., 2020b. Integrating expertises, goals and data for digital building permits - the EUnet4DBP. In: 15th $3 D$ GeoInfo Conference 2020, ISPRS - International Archives of the Photogrammetry, Remote Sensing and Spatial Information Sciences, Vol. XLII-4number W15, ISPRS, pp. 53-60.

Plazza, D., Röck, M., Malacarne, G., Passer, A., Marcher, C. and Matt, D., 2019. Bim for public authorities: Basic research for the standardized implementation of bim in the building permit process. In: IOP Conference Series: Earth and Environmental Science, Vol. 323number 1, IOP Publishing, p. 012102. 